\title{
Twenty-first century botany
}

\author{
The recent International Botanical Congress in Shenzhen was the largest meeting in its history. That a gathering \\ rooted in the superficially traditional science of taxonomy is thriving in the age of genomics and biotechnology \\ shows the strength and adaptability of modern botany and botanists.
}

\begin{abstract}
What's in a name? That which we call a rose, By any other name would smell as sweet.
\end{abstract}

Romeo and Juliet Act 2: Scene II William Shakespeare

Juliet's famous lament can be seen as an argument against nomenclature; but names cannot be so easily dismissed as arbitrary combinations of syllables. Neither Romeo nor Juliet can escape their names as both 'Capulet' and 'Montague' tell of the lovers' ancestry and the history of enmity that it holds.

Names and ancestry may not have been uppermost in the minds of all the more than 6,800 attendees of the 19th International Botanical Congress (IBC) held in Shenzhen, China, at the end of July, but given the history of the meeting perhaps it should have been. If Shenzhen is numbered the nineteenth IBC then the first was held in Paris in 1900, but the true origins date back to a series of meetings in the mid-nineteenth century to standardize the naming of plants. The first of these took place in Brussels in 1864, and was organized by the Swiss botanist Alphonse de Candolle whose father, Augustin de Candolle, wrote the 1813 book Principes élémentaires de botanique, which built upon Linnaeus's work to produce a classification for plants dividing them into discrete taxa and naming them using the now familiar binomial system of genera and species.

Initially, the meetings were annual and by the 1867 Paris gathering the laws of plant nomenclature had been codified in Lois de la nomenclature botanique, sometimes referred to as the 'Paris code. This was not the unifying naming system that was envisaged by de Candolle as American and English botanists used their own systems. It was not until 1930 that a compromise was reached between the European, American and British views at an IBC in Cambridge, UK. It was also agreed that this International Code of Botanical Nomenclature (ICBN) could only be updated at an IBC, as it has been at every meeting since 1950 . Indeed, the IBC in Melbourne in 2011 updated the codes' own name to the International Code of Nomenclature for algae, fungi and plants (ICN) to better represent its scope.

Taxonomy remains an important strand in all IBCs, but modern technologies are making its practice unrecognizable from that of the drafters of the original ICBN. For instance, Doug and Pam Soltis of the University of Florida both gave plenary talks on how 'big data' and computation tools could be integrated to help understand phylogeny and the geographical distribution of phylogenetic diversity. Similarly, Jun Wen of the Smithsonian Institution discussed combining informatics and genomics into an integrated systematics. But the meeting has grown well beyond those bounds to cover all aspects of plant biology.

The number of different plants and plant groups under investigation in work presented at Shenzhen emphasizes that even since the Melbourne IBC, advances in genetics and genomics have brought it to a stage where it can be applied not just to a small number of 'model' plants, such as Arabidopsis thaliana, Nicotiana benthamiana or Brachypodium distachyon, but also to 'non-model' plants. The now fully sequenced pineapple (Ananas comosus) for instance, is being used to investigate CAM photosynthesis with a view to engineering more drought- and heat-resistant crops; Ralf Bock talked about increasing the potential for genetic engineering by employing intra- and inter-specific gene transfer following grafting as an alternative to agrobacterium as a route to transformation; and there were sessions devoted to plants as diverse as mangroves, heathers, lilies, orchids, ferns aroids, and even a satellite session devoted to the tung tree (Vernicia fordii).

Little typifies the way in which genomics is transforming botany better than the ambitions of the China National GeneBank (CNGB) to sequence 10,000 or more plant genomes within 5 years. The CNGB opened in September of last year with support of the Beijing Genomics Institute and is conveniently placed in Dapeng, less than an hour's drive from the Shenzhen Convention Centre. Somewhat over 200 delegates attended the CNGB satellite meeting styled as 'An Invitation to Sequence 10K (or more) Plant Genomes. This follows on from the 1,000 plant transcriptome project (www.onekp. com), which has been running since 2012 and produced more than 50 published papers so far. Attempting to assemble 10,000 genomes is orders of magnitude larger than sequencing 1,000 transcriptomes and will require the development of new tools to cope with the amount of sequence data that will be produced.
The IBC continues to be a forwardlooking event. This was crystallized in China by the release of a draft 'Shenzhen Declaration on Plant Sciences' (http://www.ibc2017.cn/ Declaration). This is a manifesto for plant science in the face of the interlocking political, social and environmental challenges posed by climate change and a rapidly expanding global population. Challenges that cannot be addressed without the involvement of plant biologists. Authored by 14 very prominent researchers from China, America and Britain, it sets 7 priorities:

(1) To become responsible scientists and research communities in a changing world

(2) To increase support for the plant sciences in achieving global sustainability

(3) To cooperate across nations, regions, disciplines and cultures for common goals

(4) To develop new technologies to explore nature

(5) To accelerate the cataloguing of life on Earth for the intelligent use of nature

(6) To value and protect traditional and local knowledge of plants and nature

(7) To combine the power of the public with the power of plants through greater participation

The declaration ends with the hope that "by working together, we can unite innovative plant sciences with the needs and strengths of human societies, helping to create new paths to a green, sustainable future for Earth, with plants and people in harmony".

The IBC arose from a desire to systematize knowledge about plant life so that botanists could effectively exchange and discuss their discoveries, thereby reaching a better understanding of the world around them. Those initial goals continue to be at the heart of the IBC's activities and are only made more relevant by the realization over the last 200 years of the central role that the knowledge of plants must play to ensure a sustainable future for humanity.

The Shenzhen Congress was one of the most eagerly anticipated meetings of the last few years. The 2023 IBC in Rio has a lot to live up to.

Published online: 6 September 2017 DOI: $10.1038 / \mathrm{s} 41477-017-0018-4$ 